\title{
Kaler, Amy. (2017). Baby Trouble in the Last Best West: Making New People in Alberta, 1905-1939. Toronto: University of Toronto Press.
}

\author{
Reviewed by: Chloe Formanek, MacEwan University
}

Amy Kaler's book, "Baby Trouble in the Last Best West", combines literature from sociological and historical archival sources by examining old letters, case files, censuses, and newspaper reports which deal with issues pertaining to reproduction and childbirth in early twentieth-century Alberta. In her synthesis, Kaler focuses on the "imaginative economy of reproduction", by integrating individual and societal perspectives on the complexity of reproduction and childbirth. Perspectives vary from those who argued that childbirth was a necessity for a nation to survive to those who argued that childbirth led to various political and social problems. In doing so, Kaler questions the lens of reproduction by focusing on the analysis of childbirth, gender politics, medicalization, social programs, and mortality rates in early twentieth-century Alberta. To differentiate among reproduction and childbirth experiences across time and cultures, Kaler focused on specific social and political problems that affected both the individual and society. The book is organized into chapters that tie together the underlying theme that "making new people" may be troublesome but is essential for the protection and survival of a nation.

Kaler defines childbirth as a "social and political construct" and explains that in the early twentieth-century reproduction and childbirth was viewed by the Alberta government as a woman's most valuable asset. Further, the Alberta government viewed childbirth as a desirable 
and precious need to make Canada a stronger society (p. 39). Through historical examples, Kaler described how childbirth was influenced primarily by the government as a need to achieve resources, create good citizens, and believed it was a prominent way to grow Alberta's population within (pp. 109-110). One example Kaler provides is that babies were perceived as prizes to the government, and that expectant mothers were labeled as powerful political symbols that held the utmost responsibility for the survival of a society (p. 23).

On the other hand, Kaler explains that the Alberta government highly relied on women for reproduction, but in certain circumstances childbirth and expectant mothers became problematic for the government. For example, woman who were not capable of reproducing or women who gave birth and did not have sufficient needs to provide for themselves or their children were viewed as problematic to the state (p. 108). Further, Kaler explains that women who could not reproduce resulted in a huge loss for population growth in Alberta and became problematic to Canadian society as a whole (p. 153).

In addition to exploring childbirth as a societal collective, Kaler closely examined gender politics and its role on reproduction in early twentieth-century Alberta. To study the value of reproduction, Kaler compared gender differences between the roles that women and men played in society during this time. The idea that women were the ones in charge of babies and children, and that the deaths of these precious lives were solely on them. Further, females were the main focus of reproduction and males controlled the political and economic aspects of reproduction (p. 10). The main idea was that reproduction depended on women being good mothers not on men parenting (p. 96). Through the comparisons of a woman's role and a man's role in society, Kaler 
examined that females were significant in reproduction, but that they had no say in any political affairs that focused on reproduction and childbirth. Further due to these gender differences, Kaler found extreme inequalities and injustices between the men and women living in Alberta in the early twentieth-century.

Another key focus of reproduction in early twentieth-century Alberta, was the concept of medicalization. Essentially, Kaler deeply examined the social problems of medical systems within reproduction and childbirth. She referred to Alberta as having limited health care for expectant mothers and their children. Further, the government did not provide necessary medication, doctors, midwives, or prenatal care. Due to historical research, Kaler argued that if expectant mothers would have received efficient support and care, there would have been lower rates of maternal and infant deaths, which would have resulted in more babies being safely born into Alberta's society.

On the other hand, due to insufficient support and prenatal care, Kaler argued that the eugenics movement was created by the government as a solution to the social problem of limited health care. Further, it was the idea that the government strongly believed that getting rid of unwanted babies through sterilization would protect future mothers, and overall would protect the precious resources of the state (p. 85).

Due to the lack of medicalization and the eugenics movement, Kaler argued that they brought about the creation of social welfare programs for expectant mothers and their children. In such societies, where they lacked health care practices, groups such as the UFWA (United Farm Women of Alberta) began to fight for medical rights and maternal care for women and their children. Further, this led to the development of programs such as social welfare, adoption, and 
government funded maternity homes that allowed expectant mothers and children to receive well needed care. In addition, Kaler argued that these programs were societally beneficial in that they provided a place for women and children to go which resulted in less economical strain on the Alberta government (p. 83).

To study the value of expectant mothers and babies, Kaler focused on the effects of maternal and infant mortality on Alberta's society. The overall idea of infant death was that it led to collective problems within society, due to the idea that infant mortality resulted in a loss for expectant mothers and a loss of a precious resource. Further, the more infant deaths that occurred the more Alberta's society suffered. Also, Kaler focused on maternal mortality and argued that the death of expectant mothers was perceived as a significant sacrifice to the nation. The idea that the death of expectant mothers was symbolic to Alberta's nation because their death was significant in gifting Alberta's society with new life (p. 29).

Kaler concludes by offering her interpretation of the literature reviewed throughout the book tying everything ultimately to the making of new people. Her conclusion reads in part:

The fundamental reason for baby trouble is that social relations that organize reproduction are decisively asymmetrical. Reproduction is treated as the moral terrain of women, as the contested responsibility of men (individually and collectively), and as a precarious necessity for the state. Power, legitimacy, and responsibility are distributed unevenly across these three entities, distorting the social imaginary of reproduction... (p. 160).

Complete with numerous reports, letters, files, censuses, and illustrative examples, all detailing the interplay of reproduction that shapes women, men, and children, Kaler's book accomplishes two main goals. First, it offers an "insight into the social and political construct of childbirth and reproduction and makes a case for the idea that making new people is central to the 
work of building a society" (p. 160). Second, the book highlights the societal and political problems for women, men, and children both individually and collectively, and how these problems lead to government interventions or changes that can affect an entire nation. Kaler accomplishes these goals by drawing on previous historical research from select literature that identifies the underlying forces of childbirth and reproduction in early twentieth-century Alberta.

Kaler's intended readership, is namely sociologists, teachers, and historians who would find this book to be useful in introducing readers to the experiences of reproduction and childbirth while living in twentieth century Alberta. I believe that Amy Kaler's book is an excellent source of material that allows societies to experience first-hand the reproductive and childbearing troubles that women went through in early twentieth-century Alberta. Further, I believe that it is a good resource for University students of any discipline who are interested in Alberta's history of reproduction and childbirth.

Kaler aims to incorporate various forms of sociological, historical, political and feminist work on reproduction and childbirth in hopes of promoting past and future research on the topic. I argue that the book was too focused on women and did not include enough historical information on men when it came to their roles in reproduction. Also, the book did not provide any detail on Aboriginal and disabled children. Also, I found the introduction to be quite lengthy and the conclusion to be too short. I found "Baby Trouble in the Last Best West" interesting, informative and extremely engaging, I would recommend it to anyone who is interested in sociology or history of reproduction in the twentieth century of Alberta. 